\title{
Coordination in multiagent systems and Laplacian spectra of digraphs
}

\author{
P. Yu. Chebotarev and R. P. Agaev \\ Trapeznikov Institute of Control Sciences, Moscow, Russia \\ Received July 21, 2008
}

\begin{abstract}
Constructing and studying distributed control systems requires the analysis of the Laplacian spectra and the forest structure of directed graphs. In this paper, we present some basic results of this analysis partially obtained by the present authors. We also discuss the application of these results published earlier to decentralized control and touch upon some problems of spectral graph theory.

PACS numbers: 87.19.lr, 02.10.Yn
\end{abstract}

\section{INTRODUCTION}

In the cooperative control of distributed multiagent systems, the generation of control actions is decentralized. The actions result from negotiations between agents. As a metaphor, we can remember [1] that the musicians of "Persimfans"1], which existed in Moscow between 1922 and 1932, performed extremely complex musical compositions without a conductor. The string section formed a full circle (partly with their backs to the audience), while the wind section was situated inside of that circle. Every musician not only heard, but was also able to see the others. This way the magic chemistry among the performers, harmony and dynamic coordination between all the participants brought such a synchronization, that it became the substitute of the role of the conductor. Amazingly, one of the Persimfans' distinguishing features was their ability, according to the testimony of the extremely demanding and tough critics, to maintain a particularly subtle and profoundly individual approach to the interpretation of musical pieces, normally unthinkable without the help of the conductor.

The theory of decentralized control has a long history. In addition to the works discussed in [1], one can remember the theory of statistical consensus by DeGroot [2], methods of stepby-step coordination of expert judgements, such as Delphi methodology developed at the RAND Corporation in the 1950s [3], works on distributed networked computations and distributed decisionmaking $[4,5]$ and, of course, modeling the collective behavior of animals (see, e.g., [6]). Starting from 2003 (approximately) we can observe an avalanche of publications on decentralized control connected with the application of spectral graph theory in this field.

In this paper, we discuss continuous and discrete models of distributed coordination which can be considered as basic models of decentralized control. It is shown how the recent advantages in the algebraic graph theory (including some results published by the present authors before the "boom" imploded in 2003) can be applied in this area.

\footnotetext{
${ }^{1}$ Abbreviation for Perviy Simfonicheskiy Ansambl' (First Symphony Ensemble).
} 


\section{A CONTINUOUS MODEL OF DISTRIBUTED CONSENSUS}

Consider the basic continuous distributed consensus algorithm:

$$
\dot{x}_{i}(t)=-\sum_{j=1}^{n} a_{i j}(t)\left(x_{i}(t)-x_{j}(t)\right), \quad i=1, \ldots, n .
$$

Here, $n$ is the number of agents, $x_{i}(t)$ is an information state (characteristic, parameter, etc.) of the $i$ th agent, $a_{i j}(t) \geq 0$ is the weight with which agent $i$ takes into account the discrepancy in the information state with agent $j$. The information states can be, among others, positions (if the agents need to rendezvous in space), velocities (if they execute decentralized formation maneuvers), arrival times (if these must be synchronized), and so on.

Decentralized control usually requires solving more complex problems than simply reaching a consensus. For example, if moving in formation is considered, then a typical task is moving along a prescribed course and in a prescribed and fixed configuration. During a violent maneuver the configuration can be altered, but after the maneuver it must be restored.

Alteration and restoration of a prescribed geometric shape are also typical when a "flock" of moving physical objects encounters an obstacle or a hazard. It should be noted that reaching consensus is an important element of control strategy in all such cases. Actually, it is a key element, because it usually determines the stability of the system, its controllability, etc. That is why the analysis and synthesis of consensus algorithms, such as (1), is a necessary component of solving various problems of decentralized control. In this paper we focus on graph theoretic results underlying the analysis of distributed consensus algorithms.

Let $\Gamma(t)$ be the communication digraph associated with the consensus model (1). The vertices of $\Gamma(t)$ are identified with the agents, and $\Gamma(t)$ has an arc from vertex $j$ to vertex $i$ (denoted by $(j, i)$ or $j \rightarrow i$ ) if and only if $a_{i j}(t) \neq 0$. The presence of this arc in $\Gamma(t)$ means that agent $i$ coordinates its information state with that of $j$. The weight of the $(j, i)$ arc is $a_{i j}(t)$.

In matrix form, model (1) can be written as

$$
\dot{x}(t)=-L(t) x(t),
$$

where $x(t)=\left(x_{1}(t), \ldots, x_{n}(t)\right)^{\mathrm{T}}$ and the matrix $L(t)=\left[\ell_{i j}(t)\right]_{n \times n}$ is defined as follows:

$$
\ell_{i j}(t)= \begin{cases}-a_{i j}(t), & j \neq i, \\ \sum_{k \neq i} a_{i k}(t), & j=i .\end{cases}
$$

$L(t)$ is the Kirchhoff matrix of the communication digraph $\Gamma(t)$. Sometimes, instead of $\Gamma(t)$, the digraph $\Gamma^{\prime}(t)$ is considered such that $j \neq i$ and $a_{i j}(t) \neq 0$ result in the presence of the $i \rightarrow j$ arc (instead of $j \rightarrow i$ in $\Gamma(t)$ ). With respect to $\Gamma^{\prime}(t)$, the matrix $L(t)$ defined above is the Laplacian matrix. The classes of Kirchhoff matrices and Laplacian matrices coincide; they only differ in the way they are assigned to digraphs. We will call $L(t)$ the Laplacian matrix of the algorithm (1).

The process (1) is said to be convergent if for any initial conditions $x_{i}(0), i=1, \ldots, n$, and every $i, j=1, \ldots, n$, it holds that $\left|x_{i}(t)-x_{j}(t)\right| \rightarrow 0$ as $t \rightarrow \infty$.

The convergence properties of the algorithm (1) are determined by the spectral properties of the matrix $L(t)$.

\section{SIMPLE PROPERTIES OF THE LAPLACIAN MATRICES}

By definition, $L(t)$ has zero row sums, consequently, it is singular and the vector $\mathbf{1}=[1, \ldots, 1]^{\mathrm{T}}$ belongs to its kernel. Since the off-diagonal entries of $L(t)$ are nonpositive, the diagonal entries 
being nonnegative, and $L(t)$ has a weakly dominant diagonal, by Geršgorin's, theorem the real parts of all nonzero eigenvalues of $L(t)$ are strictly positive. That is why all nonzero eigenvalues of the matrix $(-1) \cdot L(t)$ in $(2)$ have strictly negative real parts.

The Laplacian matrix of an undirected graph is symmetric, positive semidefinite, and its spectrum is real and nonnegative. Moreover, 0 is a simple eigenvalue of this matrix if and only if the corresponding graph is connected (see, e.g., [7]). If $0=\lambda_{1} \leq \lambda_{2} \leq \ldots \leq \lambda_{n}$ are the Laplacian eigenvalues of an undirected graph, then $\lambda_{2}$ is referred to as the algebraic connectivity of the graph. This concept was introduced by M. Fiedler, and it is widely used in both theoretical studies and applications.

The Laplacian matrices of undirected graphs have been much studied. They date back to the famous matrix tree theorem by Kirchhoff; A.K. Kelmans pioneered their systematic investigation in the 1960s and 1970s; in the 1990s, the results of their study were presented in a series of reviews by R. Merris ([7] is one of them) and B. Mohar; then the monographs [8,9] were published.

The Laplacian matrices of directed graphs and relationships between their properties and the properties of the corresponding digraphs are still very poorly studied. To some extent, this is due to the fact that the mathematical problems which involve the complex spectra of digraph Laplacians are much more difficult than the corresponding problems regarding the real spectra of ordinary graphs. At the same time, the need for such studies is well recognized. Distributed control is one of the applications where this need is particularly urgent.

\section{THE CONVERGENCE OF CONSENSUS ALGORITHMS}

Assume that the Laplacian matrix $L(t)$ is kept constant: $L(t)=L$. It is not difficult to formulate a matrix-theoretic necessary and sufficient condition of convergence for the algorithm (2). As already mentioned, the vector $\mathbf{1}=[1, \ldots, 1]^{\mathrm{T}}$ belongs to the kernel of $L$. If 0 is a simple eigenvalue of $L$, then $x(t) \rightarrow \bar{x} \mathbf{1}$, where $\bar{x}$ is a scalar, consequently, $\left|x_{i}(t)-x_{j}(t)\right| \rightarrow 0$ as $t \rightarrow \infty$ for all $i, j=1, \ldots, n$, and the coordination trajectories converge. As shown in [10, Theorem 4], the zero eigenvalue of $L$ is always semisimple, i. e., its algebraic and geometric multiplicities coincide. Hence, if this eigenvalue is not simple, then the kernel of $L$ is not one-dimensional, and the convergence is violated.

Thus, the convergence analysis of algorithm (2) reduces to the determination of conditions under which 0 is a simple eigenvalue of $L$.

\section{RANKS OF LAPLACIAN MATRICES AND A CONVERGENCE CRITERION FOR DISTRIBUTED CONSENSUS ALGORITHMS}

The rank of the digraph Laplacian was studied in [11].

Recall some graph theory notation. A digraph is called strongly connected (or strong) if it contains directed paths from every vertex into every other vertex. Every maximal (by inclusion) strong subgraph of a given digraph is called its strong component or bicomponent. A basis bicomponent of a digraph is a bicomponent such that the digraph does not have any arcs flowing into this bicomponent from outside. It is easy to verify that every vertex of a digraph is reachable by a path from at least one basis bicomponent. If a basis bicomponent consists of a single vertex, we call it an undominated vertex of the digraph. A digraph is weakly connected if the graph obtained from it by replacing all arcs with undirected edges is connected. Weak components of a digraph are the maximal by inclusion weakly connected subgraphs of this digraph. A digraph is unilaterally connected if for any vertices $i$ and $j \neq i$, it contains either a directed path from $i$ into $j$ or a directed path from $j$ into $i$ (or both). Unilateral components of a digraph are its maximal by inclusion unilaterally connected subgraphs. It should be noted that the relation of unilateral connectivity need 
not be transitive, so it does not induce a decomposition of the vertex set into equivalence classes. Therefore, as distinct from the strong and weak components, unilateral components can overlap.

A subgraph of a digraph is spanning if the vertex sets of the graph and subgraph coincide. A diverging tree is a rooted directed tree containing directed paths from the root into all other vertices. A diverging forest is a rooted directed forest all of whose weak components are diverging trees.

For an arbitrary digraph $\Gamma$, consider its spanning diverging forests. Such forests are also called out-forests of $\Gamma$. A spanning diverging forest $F$ of $\Gamma$ is a maximum out-forest if $\Gamma$ has no out-forest with the number of arcs greater than in $F$. It is easily seen that every maximum out-forest has the minimum possible number of weak components (diverging trees); this number will be called the out-forest dimension of $\Gamma$ and denoted by $d$. The number of arcs in any maximum out-forest is obviously $n-d$, where $n$ is the number of vertices in $\Gamma$. The following results were obtained in [11].

Proposition 1. Let $L$ be the Kirchhoff matrix of a digraph $\Gamma$. Then rank $L=n-d$, where $n$ is the number of vertices in $\Gamma$ and $d$ is the out-forest dimension of $\Gamma$.

Proposition 2. The out-forest dimension of a digraph is equal to its number of basis bicomponents.

Proposition 3. The out-forest dimension of a strong digraph is unity.

Proposition 4. The out-forest dimension of a digraph is no less than its number of weak components and does not exceed the number of its strong components and the number of its unilateral components.

Zero is a simple eigenvalue of $L$ if and only if $\operatorname{rank} L=n-1$. That is why the above propositions imply Corollary 1.

Corollary 1. Let $L$ be the Kirchhoff matrix of a digraph $\Gamma$. Then 0 is a simple eigenvalue of $L$ if and only if $\Gamma$ has a spanning diverging tree or, equivalently, $\Gamma$ has only one basis bicomponent.

By Propositions 1 and 3, the premise of Corollary 1 is satisfied, for instance, for any strong digraph. Later on, the first statement of Corollary 1 was obtained in [12-16].

Corollary 1 provides a convergence criterion for the consensus algorithm (2).

Theorem 1. The consensus algorithm (2) with a stable Laplacian matrix $L(t)=L$ converges to a vector with equal components for any vector of initial conditions $x(0)$ if and only if the corresponding communication digraph $\Gamma$ has a spanning diverging tree or, equivalently, has a unique basis bicomponent.

A more general problem is describing the whole domain in the space of initial conditions belonging to which guarantees the fulfillment of the $\left|x_{i}(t)-x_{j}(t)\right| \rightarrow 0, i, j=1, \ldots, n$, condition, provided that convergence does not generally hold. This problem reduces to the analysis of the kernel of $L$.

\section{THE KERNEL AND EIGENPROJECTION OF THE DIGRAPH LAPLACIAN}

The eigenprojection 2 corresponding to eigenvalue 0 or, for short, simply eigenprojection of a square matrix $A$ is a projection (i. e., an idempotent matrix) $Z$ such that $R(Z)=N\left(A^{\nu}\right)$ and

\footnotetext{
${ }^{2}$ It is also called principal idempotent.
} 
$N(Z)=R\left(A^{\nu}\right)$, where $R(A)$ and $N(A)$ are the range and the kernel (null space) of $A$, respectively, and $\nu=$ ind $A$ is the index of $A$, i. e., the minimum $k \in\{0,1, \ldots\}$ such that $\operatorname{rank} A^{k+1}=\operatorname{rank} A^{k}$. In other words, the eigenprojection of $A$ is the projection on $N\left(A^{\nu}\right)$ along $R\left(A^{\nu}\right)$. The eigenprojection is unique, as an idempotent matrix is uniquely determined by its range and kernel. A number of equivalent definitions of eigenprojection can be found in [17].

In [11] we considered the normalized matrix of maximum out-forests of a digraph denoted by $\bar{J}$ (the definition of this matrix is given below in Section 8) and proved the following properties of $\bar{J}$.

Proposition 5. Let L, $d$ and $\bar{J}$ be the Kirchhoff matrix, the out-forest dimension and the normalized matrix of maximum out-forests of digraph $\Gamma$, respectively. Then $\operatorname{rank} \bar{J}=d ; \bar{J}^{2}=\bar{J}$; $L \bar{J}=\bar{J} L=0$.

By the second statement of Proposition $5, \bar{J}$ is a projection. By the third statement, $R(\bar{J}) \subseteq$ $N(L)$ and $R(L) \subseteq N(\bar{J})$. Using the relationship between the ranks of $\bar{J}$ and $L(\operatorname{rank} \bar{J}=d$ and $\operatorname{rank} L=n-d$ by Proposition 1) and the semisimplicity of 0 as the eigenvalue of $L$, we obtain that the above inclusions can be replaced with equalities. Finally, the semisimplicity of 0 as the eigenvalue of $L$ implies that ind $L=1$. Thus, the following two propositions hold true.

Proposition 6. Let $L$ and $\bar{J}$ be the Kirchhoff matrix and the normalized matrix of maximum out-forests of $\Gamma$, respectively. Then $\bar{J}$ is the eigenprojection of $L$.

Proposition 7. The linear spans of the columns and the rows of $\bar{J}$ coincide with the kernel and the left null space of $L$, respectively.

By virtue of Propositions 6 and 7, matrix $\bar{J}$ is useful for the analysis of continuous distributed consensus algorithms. In Section 7 , we will see that $\bar{J}$ is also applicable to the study of iterative consensus algorithms.

\section{ITERATIVE CONSENSUS ALGORITHMS}

Consider the finite-difference counterpart of the continuous consensus algorithm (1) with constant coefficients $a_{i j}$ :

$$
x_{i}(k+1)=x_{i}(k)-\varepsilon \sum_{j=1}^{n} a_{i j}\left(x_{i}(k)-x_{j}(k)\right), \quad i=1, \ldots, n,
$$

where $k$ is the discrete time and $\varepsilon>0$ is the step size. Rewrite algorithm (4) in matrix form:

$$
x(k+1)=P x(k),
$$

where

$$
P=I-\varepsilon L \text {. }
$$

If the step size $\varepsilon$ is small enough, then $P$ is a row stochastic matrix. This follows from the fact that $L$ has nonpositive off-diagonal entries and zero row sums. The corresponding condition of the "smallness" of $\varepsilon$ is as follows [11]:

$$
0<\varepsilon \leq\left(\max _{i} \sum_{j \neq i} a_{i j}\right)^{-1}
$$


The matrix $P=\exp (-\varepsilon L)$ corresponds to the continuous consensus algorithm (2), and (6) can be considered as the expansion of $\exp (-\varepsilon L)$ to the linear term. Matrix (6) is sometimes called the Perron matrix with parameter $\varepsilon$ of digraph $\Gamma$.

From (5), for any natural $m$ one has

$$
x(m)=P^{m} x(0),
$$

therefore, the properties of the process (5) are determined by the properties of the sequence $\left\{P, P^{2}, \ldots, P^{m}, \ldots\right\}$. From the theory of Markov chains it is known that this sequence need not converge (the necessary and sufficient condition of its convergence is the aperiodicity of the chain), but the Cesàro limit (also called the time average limit)

$$
P^{\infty}=\lim _{m \rightarrow \infty} \frac{1}{m} \sum_{i=1}^{m} P^{i}
$$

always exists and coincides with the limit of the sequence $\left\{P, P^{2}, \ldots, P^{m}, \ldots\right\}$ in case the latter converges. Otherwise, if the chain is periodic with period $s$, then

$$
P^{\infty}=\frac{1}{s}\left(P^{(1)}+\ldots+P^{(s)}\right)
$$

where $P^{(1)}, \ldots, P^{(s)}$ are the limits of its converging subsequences: $P^{(i)}=\lim _{j \rightarrow \infty} P^{j s+i}$.

It was shown in [18] that $P^{\infty}$ is the eigenprojection of $I-P$. Hence, by (6), $P^{\infty}$ is the eigenprojection of $L$ as well. This fact along with the uniqueness of the eigenprojection and Proposition 6 imply Proposition 8.

Proposition 8. Let a row stochastic matrix $P$ be connected with the Kirchhoff matrix $L$ of a digraph $\Gamma$ by equation (6) with $\varepsilon>0$. Then the matrix $P^{\infty}$ defined by (9) coincides with the normalized matrix $\bar{J}$ of maximum out-forests of $\Gamma$.

Thus, the matrix $P^{\infty}$, which determines the asymptotic behavior of the consensus algorithm (4), is equal to the normalized matrix of maximum out-forests of the communication digraph that corresponds to this algorithm. The matrix $\bar{J}$ is thereby important for the analysis of iterative consensus processes. Indeed, according to (8), to know the average asymptotic state of the algorithm (4), it suffices to consider the product $P^{\infty} x(0)=\bar{J} x(0)$. To compute the matrix $\bar{J}$, one can use the algorithm proposed in $[19,20]$, which reduces to $\min \{n-d-1,0\}$ multiplications of matrices of order $n$.

It should be remarked that Proposition 8 coincides with the Markov chain tree theorem first obtained by Wentzell and Freidlin [21] and rediscovered by Leighton and Rivest [22].

Consider the convergence of the consensus algorithm (4). Since the spectral radius of $P$ is 1 , the convergence is violated only if $P$ has an eigenvector not proportional to $\mathbf{1}=[1, \ldots, 1]^{\mathrm{T}}$ and corresponding to an eigenvalue of modulus 1 . The subspace of invariant vectors of $P$ coincides with the kernel of $L$, hence it is one-dimensional if and only if the premise of Theorem 1 holds. As for the complex eigenvalues of modulus $1, P$ can have them only if $\varepsilon$ coincides with the right endpoint of the interval (7). Indeed, in the opposite case, the increase of $\varepsilon$ up to the endpoint of the interval would have resulted in the appearance of an eigenvalue with the modulus greater than 1 , in contradiction with the stochasticity of $P$ (see also [23]).

Consequently, the convergence of consensus algorithm (4) is guaranteed by the fulfillment of the premise of Theorem 1 together with the strict form of inequality (7). Otherwise, if $\varepsilon$ coincides with the right endpoint of the interval (7) and the Markov chain determined by the matrix $P=I-\varepsilon L$ is periodic, then the convergence is violated. 


\section{THE NORMALIZED MATRIX OF MAXIMUM OUT-FORESTS}

Can the entries of the matrix $\bar{J}$, which proves to be useful for the analysis of consensus algorithms, be interpreted in terms of the communication digraph $\Gamma$ ? Such a connection is specified by the very definition of $\bar{J}$ : every entry $\bar{J}_{i j}$ of $\bar{J}$ is defined [11] as the ratio of the total weight 3 of $\Gamma$ 's maximum out-forests that have vertex $i$ belonging to a tree diverging from $j$ to the total weight of all maximum out-forests in $\Gamma$.

The following theorems [11] summarize the properties of $\bar{J}$.

Theorem 2. Suppose that $V(\Gamma)=\{1, \ldots, n\}$ and $E(\Gamma)$ are the vertex set and arc set of a digraph $\Gamma, K$ is the vertex set of some basis bicomponent of $\Gamma, K^{+}$is the set of vertices reachable by paths from $K$ and unreachable from the other basis bicomponents of $\Gamma, \widetilde{K}$ is the union of the vertex sets of all basis bicomponents, $d$ is the out-forest dimension of $\Gamma, \bar{J}$ is the normalized matrix of maximum out-forests of $\Gamma, \mathrm{T}$ is the set of spanning diverging trees in the restriction $\Gamma_{K}$ of $\Gamma$ to $K, \mathrm{~T}^{j}$ is the subset of $\mathrm{T}$ consisting of the trees that diverge from $j, \Gamma_{-K}$ is the spanning subgraph of $\Gamma$ with the edge set $E(\Gamma) \backslash E\left(\Gamma_{K}\right), \mathrm{P}^{K \rightarrow i}$ is the set of maximum out-forests of $\Gamma_{-K}$ in which $i$ is reachable from some vertex that belongs to $K, \varepsilon(\cdot)$ designates the weight of a set of subgraphs (equal to the sum of the weights of its elements), $\sigma_{n-d}$ is the weight of the set of maximum out-forests of $\Gamma$. Then the following statements are true:

1. $\bar{J}$ is a row stochastic matrix: $\bar{J}_{i j} \geq 0$, and $\sum_{k=1}^{n} \bar{J}_{i k}=1 \quad i, j \in V(\Gamma)$;

2. $\bar{J}_{i j} \neq 0 \Leftrightarrow(j \in \widetilde{K}$ and $i$ is reachable from $j$ in $\Gamma)$;

3. Let $j \in K$. For any $i \in V(\Gamma), \bar{J}_{i j}=\varepsilon\left(\mathrm{T}^{j}\right) \varepsilon\left(\mathrm{P}^{K \rightarrow i}\right) / \sigma_{n-d}$. Moreover, if $i \in K^{+}$, then $\bar{J}_{i j}=\bar{J}_{j j}=\varepsilon\left(\mathbf{T}^{j}\right) / \varepsilon(\mathbf{T})$;

4. $\sum_{j \in K} \bar{J}_{j j}=1$. In particular, if $j$ is an undominated vertex, then $\bar{J}_{j j}=1$; of $\bar{J}$.

5. If $j_{1}, j_{2} \in K$, then $\bar{J}_{\cdot j_{2}}=\left(\varepsilon\left(\mathrm{T}^{j_{2}}\right) / \varepsilon\left(\mathrm{T}^{j_{1}}\right)\right) \bar{J}_{\cdot j_{1}}$, where $\bar{J}_{\cdot j_{1}}$ and $\bar{J}_{\cdot j_{2}}$ are the $j_{1}$ and $j_{2}$ columns

Corollary 2 (from the statement 3 of Theorem 2).

1. The normalized matrix $\bar{J}_{K}=\left[\bar{J}_{i j}^{K}\right]$ of maximum out-forests of the digraph $\Gamma_{K}$ coincides with the principal submatrix of $\bar{J}$ corresponding to the basis bicomponent $K$.

2. If $i \in K^{+}$and $j \in K^{+} \backslash K$, then $\bar{J}$ is preserved under the variations of the weight of $(i, j)$.

The following Theorem 3 is concerned with the comparison of the entries of $\bar{J}$.

Theorem 3. In the notation of Theorem 2, let $K(k)$ be a basis bicomponent of $\Gamma$ that contains a vertex $k \in \widetilde{K}$. For all $i, j \in V(\Gamma)$ we have:

1. $\bar{J}_{i i} \geq \bar{J}_{j i}$

2. If $\bar{J}_{i i}>\bar{J}_{j i}$, then $i \in \widetilde{K}, j \notin K^{+}(i)$, therefore, $\Gamma$ contain no paths from $j$ into $i$;

3. If $\bar{J}_{i i}>\bar{J}_{j i}>0$, then $j \notin \widetilde{K}$, consequently, $j$ is not the root in any maximum out-forest of $\Gamma$;

4. If $\bar{J}_{i j}>0$, then $\bar{J}_{i i}=\bar{J}_{j i}$.

As mentioned above, these properties are useful for the analysis of the consensus algorithms (2) and (5) as well as generalizations of these algorithms, in particular, when the convergence is not guaranteed by the structure of the communication digraph. Some of the foregoing results regarding the kernel of the digraph Laplacian were also obtained in [24].

\footnotetext{
${ }^{3}$ The weight of a digraph is the product of the weights of all its arcs.
} 


\section{OTHER PROBLEMS OF DECENTRALIZED CONTROL}

The distributed consensus algorithm (2) is the simplest algorithm of decentralized control. Yet this algorithm is a basic one, because the linear operator of agreement $(-1) \cdot L(t)$ it contains is an indispensable constituent of more complex algorithms as well. Therefore, the convergence properties of these more complex algorithms are in many respects determined by the spectral properties of the corresponding Laplacian matrices and by the forest structure of the related communication digraphs.

In this section, we briefly survey some modifications of the consensus algorithm (2) and some other related problems of decentralized control.

First of all, it should be noted that the model (2) does not generally imply that the communication digraph $\Gamma(t)$ is fixed. The rejection of this assumption makes the model more realistic. Indeed, in many applications the agents primarily communicate with the closest neighbors, but those can be acquired or lost during the motion. To preserve the relative simplicity of the model, some restrictions on the structure of the communication digraph are applied. Usually, either it is supposed to be piecewise constant and a set of possible switchings is specified or the weights $a_{i j}(t)$ are defined with a permissible variation. Sometimes fluctuation of the weights is described by a probabilistic model. As a result, the analysis, in most cases, reduces to solving a series of problems with stable communication digraphs and combining the partial solutions. In some cases, the analysis involves the study of infinite products of stochastic matrices taken from a specific set [25].

Besides that, the model becomes more realistic if it allows communication delays. These delays can be associated with either information transmission or with processing messages after receipt. In this case, the model of the form (1) contains terms such as $x_{j}\left(t-\delta_{i j}\right)$, where $\delta_{i j}$ is a delay typical of the pair of agents $(i, j)$. If the delays are the same for all pairs of agents, then their presence does not essentially alter the properties of the coordination trajectories [26]. More general cases were considered in [27].

In many technical applications it is desirable to maximize the rate of convergence. An approach to solving such problems was developed in [28]. It is noteworthy that in case of undirected graphs, the problem of the best convergence is connected [29] with the maximization of the algebraic connectivity (the second smallest eigenvalue of the Laplacian matrix already mentioned in Section 3) of the communication graph. The algebraic connectivity itself can serve as a good measure of the convergence rate of the algorithm [30]. For the case of directed graphs, different generalizations of the algebraic connectivity were proposed, including the second smallest real part of the Laplacian eigenvalues [31], the second smallest modulus of the Laplacian eigenvalues, and the second smallest eigenvalue of the symmetric part $\left(L+L^{\mathrm{T}}\right) / 2$ of the Laplacian matrix [23,32]. However, in the general case of digraphs, the problem of optimizing the convergence rate is still insufficiently studied. Not even the problem of localizing the spectra of nonsymmetric Laplacian matrices (see Section 10) has been completely investigated.

Let us turn to some extensions of the model (2) that have a more complex structure. For the analysis of synchronization in systems of nonlinear oscillators and for solving some other problems, the following class of models was considered:

$$
\dot{x}_{i}=f\left(x_{i}\right)-\gamma \sum_{j=1}^{n} a_{i j}(t)\left(x_{i}-x_{j}\right), \quad i=1, \ldots, n, \quad \gamma>0 .
$$

It has been shown in [33] that in case of undirected communication graphs, the corresponding synchronization process is essentially determined by the algebraic connectivity of the graph. 
The consensus algorithm for a double-integrator dynamics has the form:

$$
\ddot{x}_{i}=-\sum_{j=1}^{n} a_{i j}(t)\left(\left(x_{i}-x_{j}\right)+\gamma\left(\dot{x}_{i}-\dot{x}_{j}\right)\right), \quad i=1, \ldots, n,
$$

where $\gamma>0$ controls velocity matching. It was shown in [34] that $\Gamma$ is of considerable importance here, however, for the algorithm to converge, as well as for the basic model (Theorem 1), the communication digraph must have a spanning diverging tree.

More general differential models of the second order were used to describe the motion of the complexes (flocks, schools, troop, swarms, etc.) of physical objects. "Flocking" requires both maintaining the pattern formed by the objects' positions (up to translation and/or rotation) and formation maneuvering [31,35]. In this case, the existence of a spanning diverging tree in the communication digraph is also a necessary condition of the effective control for many typical problems.

\section{LOCALIZING THE SPECTRA OF NONSYMMETRIC LAPLACIAN MATRICES}

For the analysis of models generalizing simple consensus models (1) or (4) it is necessary to know the whole Laplacian spectrum of the communication digraph rather then the sole multiplicity of its zero eigenvalue. Another important but rather difficult problem is recovering connections between the Laplacian spectrum of a digraph and the topological properties of the same digraph, including the "degree of cyclicity" and many others.

The problem of localizing the spectra of Laplacian matrices was partially solved in [10]. Let us consider some results of this paper.

A real square matrix of order $n$ will be called a standardized Laplacian matrix if (1) its row sums are equal to 0 and (2) its off-diagonal elements are nonpositive and do not exceed $1 / n$ in absolute value. This standardization enables one to consider results on the spectra of Laplacian matrices with various $n$ in the same framework. Standardized Laplacian matrices will be denoted by $\widetilde{L}$. If the class $G_{b}$ of weighted digraphs with positive arc weights not exceeding $b>0$ is considered and $L(\Gamma)$ is the Laplacian matrix of a weighted digraph $\Gamma \in G_{b}$ on $n$ vertices, then the standardized Laplacian matrix associated with $\Gamma$ in this class is, by definition, $\widetilde{L}(\Gamma)=(n b)^{-1} L(\Gamma)$.

Let $J \in \mathbb{R}^{n \times n}$ be the matrix with all entries $1 / n ; K=I-J$. Then $K$ is the standardized Laplacian matrix of the complete digraph with all arc weights $b$ in the class $G_{b}$. Define the matrices

$$
P=\widetilde{L}+J
$$

and

$$
\widetilde{L}_{c}=K-\widetilde{L}
$$

By (12) and (13), $P$ is a stochastic matrix, $\widetilde{L}_{c}$ being the standardized Laplacian matrix of the complementary weighted digraph $\Gamma_{c}$ in which $\left(b-\varepsilon_{i j}\right)$ is the weight of arc $(i, j), j \neq i$, provided that $\varepsilon_{i j}$ is the weight of this arc in $\Gamma$. If $\varepsilon_{i j}=b$, then $\Gamma_{c}$ has no $(i, j)$ arc and vice versa: if $\Gamma$ has no $(i, j)$ arc, then the weight of $(i, j)$ in $\Gamma_{c}$ is $b$. It follows from (12), (13) and the definition of $K$ that

$$
P=I-\widetilde{L}_{c}
$$

Consider the results [10] connecting the spectra of $\widetilde{L}, P$ and $\widetilde{L}_{c}$. 
Theorem 4. Let $\widetilde{L}$ be a standardized Laplacian matrix; let $P$ and $\widetilde{L}_{c}$ be defined by (12) and (13), respectively. Then for $\lambda \notin\{0,1\}$, the following statements are equivalent 4

$$
\text { (a) } \lambda \in \operatorname{sp} \widetilde{L} ; \quad \text { (b) } \lambda \in \operatorname{sp} P ; \quad \text { (c) } 1-\lambda \in \operatorname{sp} \widetilde{L}_{c}
$$

and these eigenvalues have the same geometric multiplicity. Furthermore, $v$ is an eigenvector of $\widetilde{L}$ corresponding to $\lambda \notin\{0,1\}$ if and only if the vector 5

$$
x=\left(I-\frac{J}{1-\lambda}\right) v
$$

is an eigenvector of $P$ corresponding to $\lambda$ and an eigenvector of $\widetilde{L}_{c}$ corresponding to $1-\lambda$.

Theorem 5. Let $f_{\widetilde{L}}(\lambda), f_{P}(\lambda)$ and $f_{\widetilde{L}_{c}}(\lambda)$ be the characteristic polynomials of $\widetilde{L}, P$ and $\widetilde{L}_{c}$, respectively. Then for all $\lambda \notin\{0,1\}$,

$$
\begin{aligned}
f_{P}(\lambda) & =\frac{\lambda-1}{\lambda} f_{\widetilde{L}}(\lambda), \\
f_{\widetilde{L}_{c}}(\lambda) & =(-1)^{n-1} \frac{\lambda}{1-\lambda} f_{\widetilde{L}}(1-\lambda) .
\end{aligned}
$$

Theorem 6. For any standardized Laplacian matrix $\widetilde{L}$ and the corresponding stochastic matrix $P$ defined by (12), $\widetilde{L}$ and $P$ are semiconvergent.

For completeness, the following theorem contains some results mentioned above.

Theorem 7. Suppose that $d$ and $d_{c}$ are the in-forest dimensions of the digraph whose standardized Laplacian matrix is $\widetilde{L}$ and the complementary digraph, respectively; $m_{A}(\lambda)$ is the algebraic multiplicity of $\lambda \in \operatorname{sp} A ; V_{A}(\lambda)$ is the eigenspace of $A$ corresponding to $\lambda$. Then

(i) $m_{\widetilde{L}}(0)=d, \quad m_{\widetilde{L}}(1)=d_{c}-1$;

(ii) $m_{P}(0)=d-1, \quad m_{P}(1)=d_{c}$;

(iii) $m_{\widetilde{L}_{c}}(1)=d-1, \quad m_{\widetilde{L}_{c}}(0)=d_{c}$ and these eigenvalues are semisimple;

(iv) if $v \in V_{\widetilde{L}^{(}}(0)$ and $K v \neq 0$, then $K v \in V_{P}(0)=V_{\widetilde{L}_{c}}(1)$; if $x \in V_{P}(1)=V_{\widetilde{L}_{c}}(0)$ and $K x \neq 0$, then $K x \in V_{\widetilde{L}^{(1)}}$.

Let $\widetilde{\Lambda}_{n}$ be the class of standardized Laplacian matrices of order $n$. We now turn to the problem of localizing the spectra of the matrices $\widetilde{L} \in \widetilde{\Lambda}_{n}$. The following results were obtained in [10].

Theorem 8. All eigenvalues of standardized Laplacian matrices of order $n$ belong to the meet of:

- two closed disks, one centered at $1 / n$, the other centered at $1-1 / n$, each having radius $1-1 / n$,

- two closed smaller angles, one bounded with the two half-lines drawn from 1 through $e^{-2 \pi \mathrm{i} / n}$ and $e^{2 \pi \mathrm{i} / n}$, the other bounded with the half-lines drawn from 0 through $e^{-(\pi 2-\pi n) \mathrm{i}}$ and $e^{(\pi 2-\pi n) \mathrm{i}}$, and

- the band $|\Im(z)| \leq \frac{1}{2 n} \cot \frac{\pi}{2 n}$.

Remark. Using the theorem by Dmitriev and Dynkin [36] on the spectra of stochastic matrices, it can be shown that sp $\widetilde{L}$ contains an eigenvalue with argument $\frac{\pi}{2}-\frac{\pi}{n}$ if and only if $\Gamma$ is a Hamiltonian cycle on $n$ vertices. In this case, such an eigenvalue $\lambda$ is unique, $|\lambda| \leq \frac{2}{n} \sin \frac{\pi}{n}$, and $\Im(\lambda) \leq \frac{1}{n} \sin \frac{2 \pi}{n}$.

\footnotetext{
${ }^{4} \operatorname{sp} A$ is the spectrum of $A$.
}

${ }^{5}$ For simplicity, we sometimes write $A / \alpha$ instead of $(1 / \alpha) A$, where $A$ is a matrix and $\alpha \neq 0$ is a complex number. 


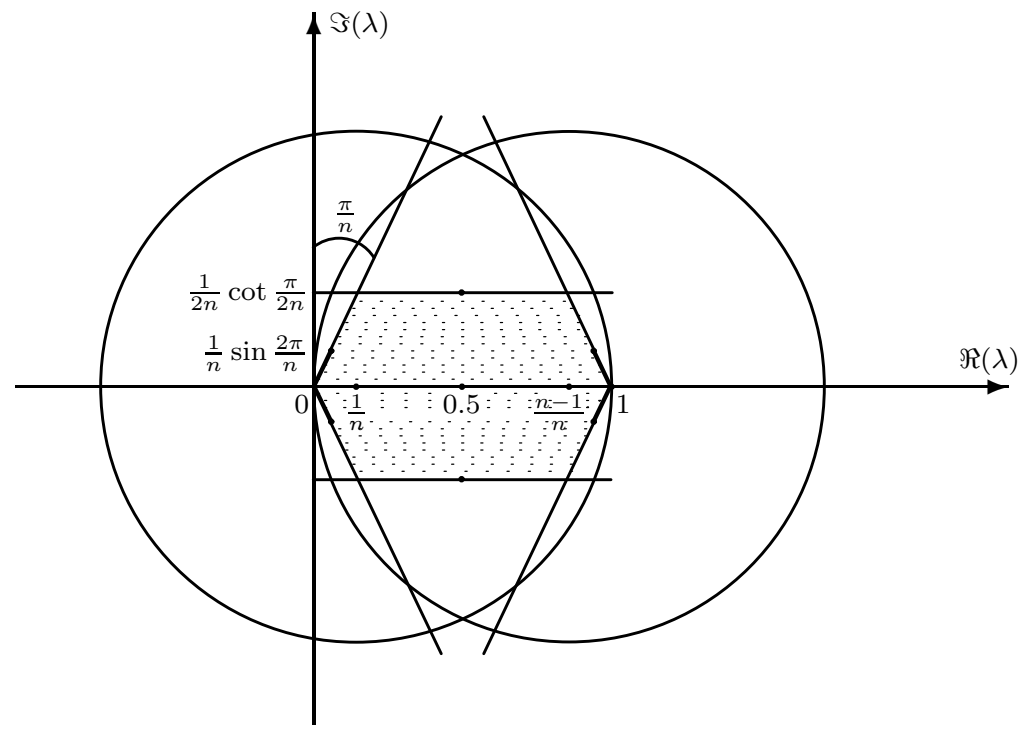

Fig. 1. The domain which, according to Theorem 8, contains the spectrum of each standardized Laplacian matrix of order $n$ (hatched); in this figure, $n=7$.

The components of any corresponding eigenvector are the vertices of a regular polygon. Similarly, sp $\widetilde{L}$ contains an eigenvalue that belongs to the segment $\left[1, e^{2 \pi \mathrm{i} / n}\right]$ if and only if the complementary digraph $\Gamma_{c}$ is a Hamiltonian cycle on $n$ vertices. As above, such an eigenvalue $\lambda^{\prime}$ is unique and $\Im\left(\lambda^{\prime}\right) \leq \frac{1}{n} \sin \frac{2 \pi}{n}$.

This remark and Theorem 8 are illustrated by Fig. 1 .

Let

$$
\begin{aligned}
\lambda_{k}(n) & =n^{-1}\left(k-e^{-2 \pi \mathrm{i} / n}-\ldots-e^{-2 k \pi \mathrm{i} / n}\right) \\
& =n^{-1}\left(k-\frac{\sin \frac{k \pi}{n}}{\sin \frac{\pi}{n}} e^{-(k+1) \pi \mathrm{i} / n}\right), \quad k=1, \ldots, n-1 ;
\end{aligned}
$$

by $S(n)$ we denote the closed convex polygon with vertices

$$
\lambda_{0}(n)=0, \quad \lambda_{1}(n), \ldots, \lambda_{n-2}(n), \quad \lambda_{n-1}(n)=1, \quad \bar{\lambda}_{n-2}(n), \ldots, \bar{\lambda}_{1}(n) .
$$

Theorem 9. Every point of the polygon $S(n)$ is an eigenvalue of some standardized Laplacian matrix $\widetilde{L} \in \widetilde{\Lambda}_{n}$.

Let $h(n)=\sup \left\{\Im(\lambda): \lambda\right.$ is an eigenvalue of some $\left.\widetilde{L} \in \widetilde{\Lambda}_{n}\right\}$.

By Theorem 8 , for all $n=2,3, \ldots$ it holds that $h(n) \leq \frac{1}{2 n} \cot \frac{\pi}{2 n}$.

Theorem 10. If $n$ is odd, then

$$
h(n)=\frac{1}{2 n} \cot \frac{\pi}{2 n},
$$

moreover, $h(n)=\Im\left(\lambda_{(n-1) / 2}(n)\right)$, where $\lambda_{(n-1) / 2}(n)$ is defined by (18). 
Proposition 9. If $n>2$ is even, then

$$
\max _{0 \leq k \leq 2 n-1} \Im\left(\lambda_{k}(n)\right)=\Im\left(\lambda_{n / 2}(n)\right)=\frac{1}{n} \cot \frac{\pi}{n}<\frac{1}{2 n} \cot \frac{\pi}{2 n} .
$$

Corollary (from Theorems 8 and 9 and Proposition 9). $\lim _{n \rightarrow \infty} h(n)=1 / \pi$.

The following conjecture is yet unproved.

Conjecture. All eigenvalues of standardized Laplacian matrices of order $n$ belong to the polygon $S(n)$ whose vertices are defined by (19).

Note that the vertices $\lambda_{k}(n)$ and $\bar{\lambda}_{k}(n)$ of the polygon $S(n)$ belong to the spectrum of the standardized Laplacian matrix $\widetilde{L}_{k}(n)=\frac{1}{n}\left(k I-C-C^{2}-\ldots-C^{k}\right)$, where $C=\left[c_{u v}\right]$ is the matrix of a cyclic permutation of order $n$ with entries $c_{u v}=\left\{\begin{array}{ll}1, & v-u \in\{1, n-1\}, \\ 0, & \text { otherwise. }\end{array}\right.$ The digraphs whose standardized Laplacian matrices are $\widetilde{L}_{k}(n)$ belong to the class of balances digraphs, which are relevant to the synthesis of multiagent control protocols (see, e.g., [23]). In balanced digraphs, the total weight of arcs converging to any vertex is equal to the total weight of arcs diverging from it. Besides that, for these digraphs, the total weight of converging arcs is the same for all vertices, i. e., they are regular.

If $n$ is even and $k<n / 2$, then of special interest are the matrices of the form $\widetilde{L}_{k}(n)+S$, where $S=\left[s_{i j}\right]$ is the standardized Laplacian matrix of a digraph obtained from the digraph with standardized Laplacian matrix $\frac{1}{n}\left(I-C^{n / 2}\right)$ by decreasing the weights of some arcs. It turns out that the maximum imaginary part of the eigenvalues of $\widetilde{L}_{k}(n)+S$ does not exceed such a part for $\widetilde{L}_{k}(n)$, and the spectrum of $\widetilde{L}_{k}(n)+S$ is invariant under any change of nonzero $s_{i j}$ and $s_{j i}$ that preserves $s_{i j}+s_{j i}$. In other words, the spectra of the standardized Laplacian matrices $\widetilde{L}_{k}(n)+S$ depend on the total weights of opposite "diagonal" arcs of the digraph rather than on their individual weights.

Theorem 11. The boundary of the polygon $S(n)$ with vertices (19) converges, as $n \rightarrow \infty$, to the curve made up by the parts of two cycloids whose parametric equations are: $z(\tau)=x(\tau)+\mathrm{i} y(\tau)$ and $z(\tau)=x(\tau)-\mathrm{i} y(\tau)$, where $\tau \in[0,2 \pi]$ and

$$
\begin{aligned}
& x(\tau)=(2 \pi)^{-1}(\tau-\sin \tau), \\
& y(\tau)=(2 \pi)^{-1}(1-\cos \tau) .
\end{aligned}
$$

Fig. 2 shows the polygons $S(n)$ at $n=4$ and $n=5$ as well as the limit curve whose equation is given by Theorem 11 .

As mentioned above, the results on localizing the spectra of Laplacian matrices given in this section are necessary for the analysis and synthesis of control algorithms that contain reaching consensus as one of their elements (see, e. g., [31]).

\section{CONCLUSION}

Recently, decentralized control of multiagent systems has become one of the most popular and rapidly evolving branches of control theory. Dozens of research groups have published many hundreds of papers and since these groups are working concurrently and are studying similar models, their results substantially overlap. To get familiar with this trend, the recent surveys and 


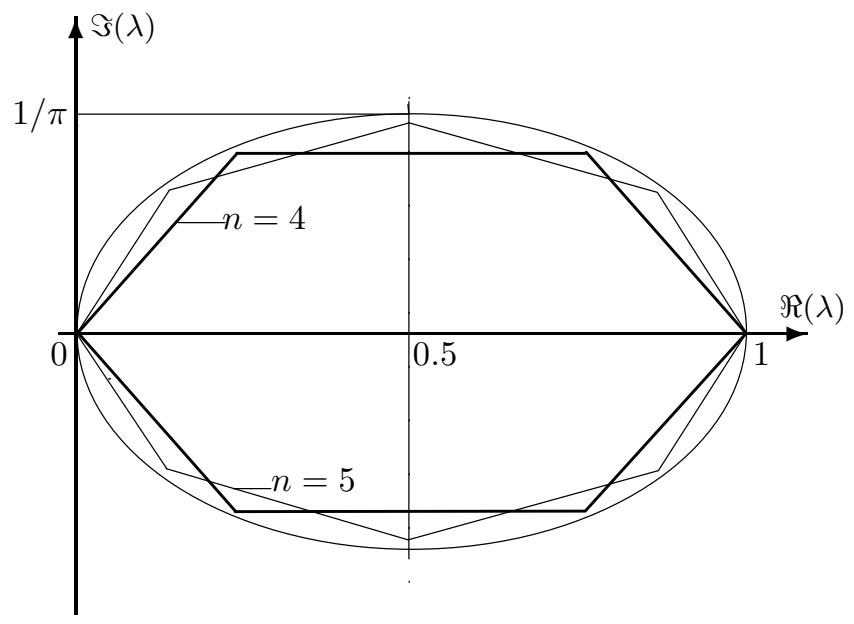

Fig. 2. The polygon of the spectra of standardized digraph Laplacians at $n=4$ and $n=5$ and the limit oval specified by Theorem 11 .

monographs $[23,32,37,38]$ can be recommended. The methods of algebraic graph theory play an increasingly important part in the studies of the last decade dealing with multiagent systems. More specifically, the subject of these investigations is the relationship between the topological properties of digraphs that represent information interchange among agents and the spectral properties of the corresponding Laplacian matrices. At the same time, the Laplacian theory of directed graph $\$ 6$ is still insufficiently developed; the need for new strong results in this field is very acute. One more thesis accepted by most researchers is that for a new stage of its development, this discipline requires more experimental studie: 7 and more practical applications of the theoretical results. These will reveal to what extent the proposed algorithms are robust to perturbations and discrepancies between theory and real behavior.

\section{REFERENCES}

1. Varshavsky, V. and Pospelov, D. Puppets without Strings, Moscow: Mir Publishers, 1988.

2. DeGroot, M.H., Reaching a Consensus, J. Amer. Statist. Associat., 1974, vol. 69, no. 345, pp. 118-121.

3. Dalkey, N.C. and Helmer, O., An Experimental Application of the Delphi Method to the Use of Experts, Management Sci., 1963, vol. 9, pp. 458-467.

4. Borkar V., and Varaiya, P., Asymptotic Agreement in Distributed Estimation, IEEE Trans. Automat. Control., 1982, vol. 27, pp. 650-655.

5. Tsitsiklis, J.N. and Athens, M., Convergence and Asymptotic Agreement in Distributed Decision Problems, IEEE Trans. Automat. Control., 1984, vol. 29, pp. 690-696.

6. Reynolds, C.W., Flocks, Herds, and Schools: A Distributed Behavioral Model, Comput. Graphics., 1987, vol. 21, no. 4, pp. 25-34.

7. Merris, R., Laplacian Matrices of Graphs: A survey, Linear Algebra Appl., 1994, vol. 197-198, pp. 143176.

8. Chung, F.R.K., Spectral Graph Theory, Providence, RI: Amer. Math. Soc., 1997.

9. Godsil, C. and Royle, G., Algebraic Graph Theory, New York: Springer-Verlag, 2001.

10. Agaev, R. and Chebotarev, P., On the Spectra of Nonsymmetric Laplacian Matrices, Linear Algebra Appl., 2005, vol. 399, pp. 157-168.

\footnotetext{
${ }^{6}$ In [31] this area was called an "unexpected new mathematical territory."

7 They are still scarce; see, e.g., [39, 40].
} 
11. Agaev, R.P. and Chebotarev, P.Yu. The Matrix of Maximum Out Forests of a Digraph and Its Applications, Automat. Remote Control, 2000, vol. 61, no. 9, part 1, pp. 1424-1450.

12. Jadbabaie, A., Lin, J., and Morse, A.S., Coordination of Groups of Mobile Autonomous Agents Using Nearest Neighbor Rules, IEEE Trans. Automat. Control, 2003, vol. 48, no. 6, pp. 988-1001.

13. Ren, W., Beard, R.W., and McLain, T.W., Coordination Variables and Consensus Building in Multiple Vehicle Systems, in Cooperative Control: A Post-Workshop Volume 2003, Block Island Workshop on Cooperative Control., Kumar, V., Leonard, N.E., and Morse, A.S., Ed., New York: Springer-Verlag, 2005, pp. 171-188.

14. Caughman, J.S., Lafferriere, G., Veerman, J.J.P, and Williams, A., Decentralized Control of Vehicle Formations, Syst. Control Lett., 2005, vol. 54, no. 9, pp. 899-910.

15. Lin, Z., Francis, B., and Maggiore, M., Necessary and Sufficient Graphical Conditions for Formation Control of Unicycles, IEEE Trans. Automat. Control, 2005, vol. 50, no. 1, pp. 121-127.

16. Moreau, L., Stability of Multiagent Systems with Time-Dependent Communication Links, IEEE Trans. Automat. Control, 2005, vol. 50, no. 2, pp. 169-182.

17. Agaev, R.P. and Chebotarev, P.Yu., On Determining the Eigenprojection and Components of a Matrix, Automat. Remote Control, 2002, vol. 63, no. 10, pp. 1537-1545.

18. Rothblum, U.G., Computation of the Eigenprojection of a Nonnegative Matrix at Its Spectral Radius, Math. Program. Study, 1976, vol. 6, pp. 188-201.

19. Agaev, R.P. and Chebotarev, P.Yu., Spanning Forests of a Digraph and Their Applications, Automat. Remote Control, 2001, vol. 62, no. 3, pp. 443-466.

20. Chebotarev, P. and Agaev, R., Forest Matrices Around the Laplacian Matrix, Linear Algebra Appl., 2002, vol. 356, pp. 253-274.

21. Wentzell, A.D. and Freidlin, M.I., On Small Random Perturbations of Dynamical Systems, Russian Mathematical Surveys, 1970, vol. 25, no. 1, pp. 1-55.

22. Leighton, T. and Rivest, R.L., The Markov Chain Tree Theorem, Computer Science Techn. Rep. MIT/LCS/TM-249, Laboratory of Computer Science, MIT, Cambridge, Mass., 1983.

23. Olfati-Saber, R., Fax, J.A., and Murray, R.M., Consensus and Cooperation in Networked Multi-Agent Systems, Proc. IEEE, 2007, vol. 95, no. 1, pp. 215-233.

24. Caughman, J.S. and Veerman, J.J.P., Kernels of Directed Graph Laplacians, Electron. J. Combinat., 2006, vol. 13, no. 1-R39.

25. Ren, W., Beard, R.W., and Kingston, D.B., Multi-Agent Kalman Consensus With Relative Uncertainty, in Proc. Amer. Control Conf., Portland, OR, 2005, pp. 1865-1870.

26. Moreau, L., Stability of Continuous-Time Distributed Consensus Algorithms, in Proc. IEEE Conf. Decision Control, Paradise Island, Bahamas, 2004, pp. 3998-4003.

27. Fang, L., and Antsaklis, P.J., On Communication Requirements for Multiagent Consensus Seeking, in Networked Embedded Sensing and Control, Lecture Notes in Control Inform. Sci., vol. 331, Antsaklis, P.J. and Tabuada, P., Ed., Berlin: Springer-Verlag, 2006, pp. 53-67.

28. Xiao, L. and Boyd, S., Fast Linear Iterations for Distributed Averaging, Syst. Control Lett., 2004, vol. 53, pp. $65-78$.

29. Kim, Y. and Mesbahi, M., On Maximizing the Second Smallest Eigenvalue of a State-Dependent Graph Laplacian, IEEE Trans. Automat. Control, 2006, vol. 51, pp. 116-120.

30. Olfati-Saber, R. and Murray, R.M., Consensus Problems in Networks of Agents with Switching Topology and Time-Delays, IEEE Trans. Automat. Control, 2004, vol. 49, pp. 1520-1533.

31. Veerman, J.J.P., Lafferriere, G., Caughman, J.S., and Williams, Flocks and Formations, J. Statist. Physics, 2005, vol. 121, no. 5-6, pp. 901-936.

32. Wu, C.W., Synchronization in Complex Networks of Nonlinear Dynamical Systems, World Scientific, 2007.

33. Preciado, V.M. and Verghese, G.C., Synchronization in Generalized Erdös-Rényi Networks of Nonlinear Oscillators, in Proc. IEEE Conf. Decision Control, Eur. Control Conf., Seville, Spain, 2005, pp. 4628 4633. 
34. Ren, W. and Atkins, E., Second-Order Consensus Protocols in Multiple Vehicle Systems with Local Interactions, in Proc. AIAA Guidance, Navigation, Control Conf., San Francisco, CA, 2005. Paper AIAA-2005-6238.

35. Lawton, J.R., Beard, R.W., and Young, B., A Decentralized Approach to Formation Maneuvers, IEEE Trans. Robot. Automat., 2003, vol. 19, no. 6, pp. 933-941.

36. Dmitriev, N. and Dynkin, E., Characteristic Roots of Stochastic Matrices, Izv. Akad. Nauk SSSR (Bull. Acad. Sci. USSR), Mat., 1946, vol. 10, pp. 167-184. Translated in Eleven Papers Translated from the Russian, Bernik, V.I., Ed., Amer. Math. Soc., 1988.

37. Ren, W., Beard, R.W., and Atkins, E.M., Information Consensus in Multivehicle Cooperative Control, IEEE Control Syst. Magazin, 2007, vol. 27, no. 2, pp. 71-82.

38. Ren, W. and Beard, R.W., Distributed Consensus in Multi-Vehicle Cooperative Control, London: Springer-Verlag, 2008.

39. Regmi, A., Sandoval, R., Byrne, R., et al., Experimental Implementation of Flocking Algorithms in Wheeled Mobile Robots, in Proc. Amer. Control Conf., Portland, OR, 2005, pp. 4917-4922.

40. Marshall, J.A., Fung, T., Broucke, M.E., et al., Experiments in Multirobot Coordination, Robot. Autonom. Syst., 2006, vol. 54, no. 3, pp. 265-275.

This paper was recommended for publication by B.T. Polyak, a member of the Editorial Board 